\title{
Advances in the understanding of throwing injuries of the shoulder
}

\author{
R G Hackney
}

Shoulder problems in throwersbiomechanics and pathology

Athletes whose sport involves an overhead throwing action include not only the javelin thrower and baseball pitcher, but also tennis players and cricketers. Swimmers use a similar action. The "overhead athlete" is prone to injuries which differ from those of the nonthrowing population.

The basic throwing action is divided in to several distinct phases. Wind-up, cocking, early acceleration, acceleration, and follow-through. The shoulder is in the middle of a chain in the throwing action that begins with the feet pushing off the ground, through the legs and trunk to the hand. Only $11-17 \%$ of the velocity of a tennis serve is produced by the shoulder alone. The shoulder must not be viewed in isolation. ${ }^{1}$

A study of the forces involved in throwing, and of how muscles controlling the shoulder function in both the normal and pathological states, is important in understanding the causation, treatment, and rehabilitation of the injured throwing athlete. A brief overview of important aspects of the biomechanics of throwing is given.

The glenohumeral joint relies upon soft tissue control for stability. The static stabilisers include the glenoid labrum and the glenohumeral ligaments. The labrum is a fibrous extension to the glenoid which serves to deepen the shallow glenoid concavity. It provides a sucking effect against the humeral head analogous to the cup on the end of a child's arrow. The labrum is the site of attachment of the glenohumeral ligaments and superiorly, the long head of biceps. The glenohumeral ligaments are a very well studied group of ligaments which control the stability of the shoulder in varying positions.

The inferior glenohumeral ligament (IGHL) is the main static support of the shoulder in the abducted and externally rotated position. The range of motion in external rotation of the thrower's shoulder is extreme. External rotation in pitchers during the cocking phase averages 178 degrees. $^{2}$

The degree of laxity in the IGHL and other anterior structures necessary to permit such a range of motion in external rotation predisposes the athlete to anterior subluxation and instability.
The rotator cuff provides active stabilisation of the shoulder throughout its range of motion. The function of the rotator cuff is to centre the humeral head in the glenoid, allowing the prime movers of the shoulder such as the deltoid, pectoralis major, and others to provide the power. The glenohumeral joint is not a perfect ball and socket joint, but glides. The humeral head will translate up to $4 \mathrm{~mm}$ in the normal shoulder. A properly functioning rotator cuff will prevent excess translation of the humeral head in both antero-posterior and superior directions.

Supraspinatus pulls the humeral head into the glenoid as part of the rotator cuff. Electromyographic (EMG) studies show that supraspinatus is most active in late cocking, when the shoulder is most susceptible to anterior translation and subluxation. Comparisons between various groups have been made. Skilled throwers use their rotator cuff muscles less than unskilled throwers, and make more use of subscapularis and latissimus dorsi, both in acceleration and deceleration. They are also able to demonstrate selective use of cuff muscles. Fatigue of supraspinatus leads to abnormal movement of the humeral, predisposing the shoulder to injury. The greater use of supraspinatus in relatively untrained throwers requires specific training of supraspinatus pre-season. ${ }^{3-5}$

Supraspinatus is only working at approximately $20-40 \%$ of maximum voluntary contraction during the later stages of the throw, acting eccentrically in follow-through.

The action of infraspinatus and teres minor is external rotation and stabilisation of the shoulder. Peak activity of these muscles occurs in late cocking and follow-through. Subscapularis activity peaks in late cocking when it contracts eccentrically, protecting the anterior capsule. ${ }^{5}$

Scapular control is increasingly recognised as being very important in the biomechanics of the shoulder, providing a stable glenoid for the action of the rotator cuff muscles. The analogy of a seal balancing a ball on its nose is a good one. Both the nose and the ball move independently. Control of the ball (the humeral head) depends upon control of the nose (the glenoid). Serratus anterior and trapezius are the two most important muscles to be considered. Serratus anterior works very strongly 
in the later stages of the throw. Glousman found the serratus anterior was consistently active in stabilising the scapula in a variety of sports. $^{4}$

Throwers with chronic anterior instability have been shown to have altered activity of all muscles about the shoulder with the exception of the deltoid. Supraspinatus activity is mildly increased throughout the throw, but infraspinatus has increased activity only in early cocking and follow-through.

Serratus anterior shows decreased activity and hence the thrower develops reduced horizontal protraction of the scapula, adding to the strain on the anterior stabilisers.

The tendon of the long head of biceps serves an important function as a depressor of the humeral head. Long head of biceps also contributes to anterior shoulder stability by increasing torsional rigidity of the shoulder, resisting the excessive external rotatory forces occurring in the vulnerable abducted and externally rotated position. Biceps has been shown to have increased firing in unstable shoulders during a throw, possibly reducing stresses upon the inferior glenohumeral ligament. $^{6}$

At the Kerlan Jobe clinic in Los Angeles, the biomechanics laboratory includes a baseball mound where pitchers throw while being monitored by six high speed video cameras and 16 lead EMGs. Muscle output is related precisely to the throwing action. Analyses such as these have produced some very impressive results. The speeds produced by a throwing action are very high indeed. Peak angular velocities of internal rotation have been measured at 9000 degrees per second. Peak accelerations reach $600000 \mathrm{deg} \mathrm{s}^{-1} \mathrm{~s}^{-1}$.

The forces involved are equally impressive; the muscular torque at the time of maximum external rotation reaches over 50 Newton metres (nm) averaging $29 \mathrm{~nm}$, equivalent to holding an $8 \mathrm{~kg}$ weight in the hand. These forces are equivalent to body weight wrenching the arm from the body. At release, 400 Newtons (n) of posterior force $1090 \mathrm{n}$ of compressive force and $98 \mathrm{~nm}$ of horizontal abduction are generated in the follow-through. ${ }^{8}$

\section{Pathology}

PATHOLOGIES SEEN IN THE THROWING ATHLETE'S SHOULDER

These comprise capsulo-labral tears, detachments or stretching, together with a partial thickness tear of the undersurface of the rotator cuff (superior labral detachments where the long head of biceps inserts into the superior glenoid labrum-SLAP lesions ${ }^{9}$ ); subacromial impingement (in the older athlete); osteolysis of the distal end of the clavicle, as seen in weight lifters ${ }^{10}$; and dead arm syndrome. ${ }^{11}$

\section{LAXITY VERSUS INSTABILITY}

Laxity, by definition, is the normal glenohumeral translation for a given individual. Instability occurs where the laxity becomes pathological and causes symptoms. This may occur with an injury or as a process in sports which demand extremes of range of motion, for example throwing, butterfly swimming.

\section{ANTERIOR INSTABILITY}

An understanding the mechanisms of injury is the key to understanding the pathology and subsequent treatment. As a general principle, an injury to one of the restraints of the shoulder, whether static or dynamic, will force an increased load to be borne by the other portions. The range of motion found in the typical thrower predisposes the athlete to anterior instability. The anterior capsular restraints are sufficiently lax to allow 180 degrees of external rotation at 90 degrees of shoulder abduction. Translation of the humeral head in lesser degrees of external rotation is then limited by the glenoid labrum as the static and the rotator cuff as the dynamic stabilisers. Unless it is symptomatic, the shoulder is lax rather than unstable. This is an important point to establish.

Tightness of the posterior capsule, a common maladaptation seen in throwers, accentuates anterior translation of the humeral head.

A long training session, a prolonged period of pitching in baseball where 100 to 130 throws may be typical per game, or excess serving in tennis may lead to fatigue and reduced function of the rotator cuff. Injury to the cuff for this or any other reason may have two effects. Firstly, the humeral head is allowed to undergo excessive translation. Anteriorly, this leads to a riding up over the glenoid labrum causing abrasion and decreased function of the labrum and further stretching of the IGHL. There may also be some superior translation which may cause subacromial impingement. However, this latter theory is the subject of some debate. In addition, cuff fatigue has been thought to lead to failure to control the shoulder in follow-through, leading to the theory of eccentric overload. Eccentric overload is said to occur when the force of the throw in followthrough wrenches the shoulder anteriorly, forces measured at close to body weight. The rotator cuff tendon tears as the stretch on the tendon is sufficient to cause micro and macro tears. This has been put forward as the cause of the tears of the rotator cuff seen on magnetic resonance imaging (MRI) and at arthroscopy.

\section{IMPINGEMENT VERSUS INSTABILITY}

Much has been written on the cause of shoulder pain in throwers, debating impingement or instability as the principal cause. It is now generally recognised that shoulder pain in the throwing athlete is caused by subtle subluxation rather than impingement. Fortunately the majority of athletes respond to a rehabilitation programme. One major reason for relegating the role of impingement is the fact that those who failed conservative treatment and underwent surgery had unacceptable results, whether by open or arthroscopic subacromial decompression. Where capsular laxity was rec- 
ognised, the results of subacromial decompression produced only a $25 \%$ return to the previous level of activity. No swimmers or pitchers returned to the same level. Treatment of rotator cuff tears found at the time of surgery made no difference to the outcome. The obvious conclusions are that neither subacromial impingement nor rotator cuff pathology are responsible for symptoms in the high demand overhead athlete. ${ }^{312}$

Subacromial impingement certainly does occur, but the balance has swung heavily towards instability as the principal cause of symptoms. The increased translation of the humeral head found in the unstable shoulder must be corrected or results of surgical treatment will be unsuccessful. Subacromial impingement is probably more common in the throwing athlete over 40 years old.

\section{INTERNAL IMPINGEMENT}

The theory of subacromial impingement and/or eccentric overload as a principal cause of the rotator cuff tears in throwing athletes has been challenged by Chris Jobe, his father Frank, and coworkers. ${ }^{13}$ They point out that the usual site of rotator cuff damage in subacromial impingement is the superior surface of the cuff, and that eccentric overload is more likely to cause a central degeneration of the cuff tendon. Subacromial decompression as a treatment of these athletes has limited success only. If eccentric overload of the cuff was taking place, then one would expect to measure very high EMG activity in the supraspinatus and infraspinatus muscles in follow-through. This is not the case.

They propose a new mechanism for the cause of the undersurface cuff damage. At arthroscopy it is possible to observe an impingement of the rotator cuff against the postero-superior glenoid labrum in the position of abduction and external rotation, equivalent to the position of the shoulder in late cocking. This is increased where posterior capsular tightness forces the impingement to occur. This so called internal impingement does seem to explain the pathology seen and accounts for the rather posteriorly positioned undersurface damage of the rotator cuff.

\section{SLAP LESIONS}

Tears of the superior labrum both anterior and posterior are termed SLAP lesions. The SLAP lesions seen in throwers are probably due to excess pull of the biceps on the glenoid labrum or a fall on the outstretched arm. The superior labral lesion is detrimental to anterior shoulder stability as it decreases the shoulder's torsional rigidity and places greater strain upon the inferior glenohumeral ligament. Damage to the IGHL increases any instability.

BICIPITAL TENDINITIS

Biceps tendinitis is rarely made as a primary diagnosis; $95-98 \%$ of cases of bicipital tendinitis are secondary to subacromial impingement or a SLAP lesion. ${ }^{14}$ Occasionally the tendon may sublux from its groove with a painful snap; this is usually treated with tenodesis.
DEAD ARM SYNDROME

Dead arm syndrome occurs when a throw is accompanied by a sudden sharp or paralysing pain. The arm goes "dead" and hangs by the side followed by an ache afterwards. Throwing distance and speed deteriorate with time. Dead arm syndrome was described in athletes who complained of subluxation of the shoulder in $1969 .^{15}$

It was first attributed to occult anterior instability of the shoulder by Rowe and Zarins. ${ }^{11}$ The shooting pain is probably caused by traction upon the brachial plexus in followthrough, the decelerators failing to control the integrity of the joint.

\section{History}

The following information is obtained from the history: the sport involved, how much throwing is involved, and how much throwing training is undertaken; when in the throw does the pain occur, in the cocking, acceleration, or followthrough? Does the athlete complain of pain instability and weakness? Ask specifically for a history of dead arm syndrome. Does the shoulder click painfully? Did the initial injury occur throwing or in other training activity?

Rotator cuff pain occurs at night. Impingement pain occurs in a position of flexion, abduction and internal rotation. The apprehension position is reproduced in bench press, press ups, or stretching when yawning!

Gleno-humeral pain is often referred to the deltoid insertion. Non-specific pain is felt anteriorly. Acromioclavicular joint pain is well localised to the joint itself.

\section{Examination}

LOOK

Look along the line of the spine of the scapula for wasting of infra and supraspinatus; swellings; scars.

MOVE

Assess range of motion, in particular look for any limitation of internal rotation in the throwing arm, tight posterior capsular structures with limited cross arm adduction. External rotation should be assessed with the arm at the side and at 90 degrees of abduction. Look for loss of scapular rhythm; any scapular dyskinesias or winging?

Assess the anterior and posterior draw signs; can the head of the humerus ride up over the rim of the glenoid? The sulcus sign indicates inferior laxity. Test for laxity in other joints using the Beighton scale.

Test power, weakness and muscular imbalances, for example, weakness of external rotation, supraspinatus in the scapular plane, shoulder retraction for subscapularis. Assess pain and weakness with resisted movements.

FEEL

The anterior joint line is palpable in a non-heavily-muscled individual, as are the tuberosities. The long head of biceps is palpable in its groove between them. When unstable, the tendon may be palpated as it flicks out of 
Key steps in rehabilitation programme

Control symptoms

Prevent cuff shutdown

Strengthen and stretching work using functional planes of motion

Dynamic stabilisation with eccentric control of the deceleration phases

Scapular control and rhythm to provide a stable scapular platform

Rhythmic stabilisation drills with motion

Core stability, conditioning the entire body

Progression to overhead functional demands, training the throwing arm

Endurance training

Periodisation and preseason conditioning the groove with rotation of the shoulder in abduction. The acromioclavicular joint may be tender.

\section{SPECIAL TESTS}

Impingement

Impingement signs: the arm is placed in the examiner's hand in 30 degrees of flexion and with 90 degrees of abduction at the shoulder, and is then internally rotated. This compresses the cuff against the roof of the shoulder and reproduces the pain of subacromial impingement. End of range pain on full forward flexion performs a similar role.

\section{Instability}

Shoulder instability in a throwing athlete is often subtle and usually does not result in frank dislocation. The athlete may be totally unaware of any problems of instability. There is a fine line between laxity and instability. The task of the examination is to assess where the athlete lies along the development of symptomatic instability and the presence of any multidirectional instability.

The classical position for testing - apprehension occurs with the shoulder in an abducted and externally rotated position.

The Jobe relocation test is a very sensitive method of detecting subtle instability. The patient lies supine with the edge of the table under the affected shoulder. The examiner places the shoulder in the classic position of abduction and external rotation. He then externally rotates the shoulder until the patient experiences pain. An asymptomatic, stable shoulder will feel uncomfortable but not particularly sore. The examiner then places his hand over the humeral neck and exerts a downward force, relieving pain. The test gets its name because this manoeuvre was thought to relocate the glenohumeral joint from an anteriorly subluxed position. Maintaining the pressure, the shoulder is externally rotated further until pain recurs. The examiner then releases the hand over the humeral neck. If the test is positive the patient experiences a sudden exacerbation of pain. Frank Jobe now suspects that rather than testing for anterior subluxation the test actually detects internal impingement, and that the examiner's hand is pushing the humeral head away from the postero-superior glenoid (Job FW, Jobe CM, personal communication).

Tests for SLAP lesions are not sensitive or specific. The best test is probably with the arm outstretched at 90 degrees abduction of the shoulder, supinated, and then resisting downwards force reproducing pain for a positive test (Altchek D, personal communication).

\section{Investigation}

Plain $x$ rays are taken as standard. These reveal abnormalities of the acromio-clavicular joint, a Hill Sachs lesion. Bony Bankart defects, where the antero-inferior glenoid is deficient, can be seen where the defect is large, but computerised tomography is better for detecting smaller lesions.

Ultrasound is reported to be effective in detecting damage to the rotator cuff in skilled hands, but the technique is very user dependent.

MRI scanning, with or without an arthrogram, is becoming the most effective noninvasive means of identifying pathology within the shoulder joint. The advent of real time MRI scanning may improve our understanding still further.

The radiologist should put the arm into external rotation to tension the anterior capsule and demonstrate more effectively the Bankart and capsular lesions.

MRI can demonstrate sublabral cysts which can spread under the cuff and cause pressure upon the suprascapular nerve, a cause of rotator cuff dysfunction.

Examination under anaesthetic is an integral part of assessing the degree of instability.

\section{Treatment}

\section{REHABILITATION}

The vast majority of throwers will respond to conservative treatment alone. The rehabilitation methods developed in the USA for baseball pitchers have become well researched and refined. The importance of scapular stability, dynamic stability of the glenohumeral joint, proprioceptive work, and the techniques of reintroducing throwing are stressed. ${ }^{16-18}$

The first step in the rehabilitation programme is control of symptoms. This can be achieved with ceasing the aggravating activity. Mobility must be maintained as much as possible and immobilisation is contraindicated. Non-steroidal anti-inflammatorydrugs, corticosteroids into the subacromial bursa, and ice, heat, ultrasound, etc can all be used to relieve pain.

\section{SPECIFIC ADAPTATION TO IMPOSED DEMANDS}

This phrase has been coined to describe the philosophy of the rehabilitation programme. Rehabilitation must provide dynamic stability, maintaining flexibility and range of motion. As a general principle, closed chain exercises result in less shear, greater compressive forces, and increased proprioceptive input and are recommended for the early stages of rehabilitation. The key steps in the rehabilitation programme are given in the table.

\section{STRETCH}

Posterior capsular tightness is a frequent finding in throwers. Stretching in internal rotation with posterior and crossed arm adduction is an early, integral part of the rehabilitation 
programme. Until the rehabilitation programme has advanced to throwing, the athlete should avoid most other stretches, specifically those increasing external rotation in abduction.

\section{STRENGTHENING}

Techniques

If necessary eliminate gravity, using short lever arms at the beginning. If the shoulder is very sore, then isometric exercises are used initially, progressing to isotonic work

Advance to use gravity, then elasticated cord and free weights. Elastic sports cords are used 15-20 minutes per day with roughly $25-30$ repetitions per exercise.

\section{Rotator cuff exercises}

Training with internal and external rotators. Eccentric work is thought to be important if the theory of eccentric overload is accepted. Certainly the awareness of position of scapula is mandatory.

Supraspinatus - Exercises to be performed in the scapular plane, that is, 30 degrees forward, in internal rotation, and below 90 degrees of abduction, eliminating gravity by lying prone at the start.

Infraspinatus and teres minor - Lying prone or on the side moving in external rotation.

Subscapularis - Supine internal rotation, and internal rotation at the side with elastic tubing, slow return will give eccentric work.

\section{Scapular strengthening}

Shoulder shrugs for trapezius, seated rowing for rhomboids and serratus, upright rows for trapezius, press-ups, plus protracting the scapula at the end of a normal press-up. Do not allow the chest to sink onto the floor as this merely recreates the position of apprehension.

\section{Pectoralis major and latissimus dorsi}

Latissimus pull downs for deceleration control; latissimus dorsi protects and unloads supraspinatus in follow-through.

\section{Deltoid}

Shoulder flexion abduction and extension.

Biceps exercises

Biceps curls and variations.

\section{ADVANCED PROPRIOCEPTIVE WORK}

A degree of imagination is necessary to invent exercises which will provide strengthening and proprioceptive work. For example, walking on hands, cross arm walking, balancing on wobble board, physio foam, physioball. The physiotherapist can provide manual resistance with elastic cord or dumbbells, and deliberately alter angles of pull, and provide sudden changes which require an immediate reaction to enhance proprioception.

More advanced circuit training work should include plyometric exercises. There are many variations on the press-up theme, for example push-ups onto a box with deceleration; eccentric work landing from it then pushing back onto the box; one handed clapping press-ups, using one knee with the physiotherapist requiring extra work by pushing the athlete, or using an unstable platform are all advanced exercises.

CRITERIA FOR RETURN TO THROWING

These are: pain-free function; normal range of motion; and isokinetic tests reaching expected normal levels.

GRADUATION TO SPORT-SPECIFIC EXERCISES

Use longer lever arms, throw light balls slowly, catch ball returning from a net or wall; ball dribbling for endurance; exercises such as flies, bench pressing with a wide seated grip; medicine ball for catching and overhead throwing.

\section{Suggested exercises using $3 \mathrm{~kg}$ medicine ball}

Trunk twisting with arms extended; placing ball on floor then overhead; throwing and catching against a trampoline; catch and throw again to same and other side, underhand, two handed; long leg sitting, sideways throw, trains the trunk and shoulder; sit-ups, throw and catch.

\section{ENDURANCE}

The fatigue model of aetiology of rotator cuff dictates that endurance training plays an important preventative role. Suggested methods include increasing the number of repetitions, wall dribbling, and general exercisers such as cross country skiing machines the versa climber and rowing machines.

\section{Surgical management}

Fortunately the majority of throwers respond to a properly managed rehabilitation programme. Failure of adequate conservative treatment is the indication for surgery. The surgeon must ensure that the rehabilitation programme has been undertaken properly.

Examination under anaesthetic (EUA) is performed in every case in varying degrees of abduction and external rotation, and comparison with the normal side made. Stability in all directions is assessed. Multidirectional subluxation is important not to miss. Tightening only one side of the shoulder in this situation is a potential disaster.

Multidirectional instability is more common than previously appreciated. The primary pathology is capsular laxity and redundancy. Surgery must be aimed at tightening of the capsule, tailoring the surgery to the individual. ${ }^{19}$

Shoulder arthroscopy follows EUA. The labrum and glenohumeral ligaments are probed and their status assessed as an important part of the diagnostic workup. The drive-through sign is positive when the arthroscope can be pushed across the glenohumeral joint from the posterior portal to the anteroinferior glenoid. This indicates capsular laxity. Many procedures can be undertaken through the arthroscope. Subacromial decompression, Bankart repairs, capsular shifts, and rotator cuff repair can all be performed with appropriate skills and instrumentation. However, these 
techniques require a long learning curve and only in expert hands do success rates approach those of open procedures.

Simple debridement of damage to the glenoid labrum produces some short term benefits, but after two to three years shoulder pain returns. ${ }^{2021}$ Reports of successful outcome have emphasised the importance of rehabilitation following surgery, which may go some way towards explaining the success! In one series $62 \%$ of baseball pitchers were unimpaired after arthroscopic debridement without gross instability or a Bankart lesion. The average age of patients in this series was 19 years, before chronic attenuation of the glenohumeral ligaments associated with prolonged throwing occurs. This separates the patients in this series from others. ${ }^{22}$

OPEN SURGICAL CORRECTION OF INSTABILITY The recurrence rates for frank recurrent dislocation are similar for the commonly performed procedures such as the Putti-platt, Bristow, etc. For the throwing athlete, the procedure that allows a maximum range of motion is essential. The Bankart type procedure where the capsule is repaired back onto the rim of the glenoid is best. $^{23}$

The hard end point of success in the baseball pitching population is a return to previous level of competition, not simply prevention of further symptoms. Given this task it is perhaps not surprising that many procedures fail to achieve great success.

Lombardo in 1976 concluded that throwers were not capable of returning to high performance levels following the Bristow procedure. Few throwing athletes are included in the studies of surgery for shoulder instability. Careful reading of the results frequently shows that the results for throwers are not as good as other athletes. ${ }^{24} 25$

The best published results come from the Kerlan-Jobe clinic in Los Angeles. The technique used is termed an anterior capsulolabral reconstruction.

\section{ANTERIOR CAPSULOLABRAL RECONSTRUCTION} The approach splits rather than detaches the subscapularis muscle to gain access to the anterior margin of the glenoid and damaged labrum. Capsular laxity is corrected by reefing a horizontal incision in the capsule from north to south. The relatively atraumatic treatment of muscle allows earlier rehabilitation. Exercises are started on day 1 . Results show 75 patients retained a full retention of range of motion and had excellent return to throwing sports. Seventy five per cent of professional baseball players and $100 \%$ of amateur throwers returned to the same level of competition. The approach gives limited view for correction of multidirectional instability and does not work as well. ${ }^{26}$

David Altchek at The Hospital for Special Surgery, New York described 42 shoulders with a capsular shift on the glenoid side and correction of the labral pathology. Ninety five per cent excellent results were achieved, with reasonable return to throwing, though with less speed of throw than preoperatively. ${ }^{27}$

ROTATOR INTERVAL

The rotator interval lies between the tendons of subscapularis and supraspinatus. In chronic anterior instability this defect can become so significant as to be a cause of anterior ligamentous insufficiency. The interval should be identified at the time of surgery. Closure of the interval with a couple of sutures is recommended.

\section{Conclusion}

The recognition of the role of instability of the shoulder as the principal cause of pain in the throwing athlete has evolved in recent years. Knowledge of the basic sciences of anatomy and biomechanics has led to a better understanding of the nature of the pathology and its causes. Advances in treatment methods, both conservative and surgical, have resulted in a greater return to activity at preinjury levels.

Steven Copeland reviewed throwing injuries in this journal in $1993 .^{28} \mathrm{He}$ described the mechanism of throwing, pathology, and the treatment of the injured shoulder in the throwing athlete. This article should be read with reference to that or a similar review on throwing injuries. Since 1993 there have been advances in the theory, understanding, and treatment of throwing injuries to the shoulder. I was recently able to benefit from a travelling fellowship from the Royal Air Force to study shoulder injuries and sport. This gave me an opportunity to visit several centres in the United States of America and discuss new theories with their originators. The aim of this paper was to discuss the new ideas.

1 Elliot BC. Biomechanics of the serve in tennis a biomedical perspective. Sports Med 1988;6:285-94.

2 Dillman CJ, Fleisig GS, Andrews JR. Biomechanics of pitching with emphasis upon shoulder kinetics. F Orthop Sports Phys Ther 1993;18:402-8.

3 Glousman RE. Instability versus impingement syndrome in the throwing athlete. In: Shoulder arthroscopy and related surgery. Orthop Clin North Am 1993;24:89-99.

4 Glousman RE. Electromyographic analysis and its role in the athletic shoulder. Clin Orthop Rel Res 1993;288:27-34.

5 Glousman R, Jobe FW, Moynes D, Antonelli D, Perry J. Dynamic electromyographic analysis of the throwing shoulder with glenohumeral instability. $f$ Bone foint Surg shoulder with glenoh

6 Rodosky MW, Harner CD, Fu FH. The role of the long head of the biceps muscle and superior glenoid labrum in anterior stability of the shoulder. Am F Sports Med 1994;22:121-30.

7 Pappas AM, Zawacki RM, Sullivan TJ. Biomechanics of baseball pitching. Am $\mathcal{f}$ Sports Med 1985;13:216-22.

8 Fleisig GS, Andrews JR, Dillman CJ, Escamilla RF. Kinetics of baseball pitching with implications about injury mechanism. Am F Sports Med 1995;23:233-9.

9 Snyder SJ, Karzel RP, Del Pizzo W. SLAP lesions of the shoulder. Arthroscopy 1990;6:274-9.

10 Scavenius M, Iverson BF. Nontraumatic clavicular osteolysis in weight lifters. Am $\mathcal{F}$ Sports Med 1992;20:463-5.

11 Rowe CR, Zarins B. Recurrent transient subluxation of the Rowe CR, Zarins B. Recurrent transient subluxatio

12 Tibone JE, Jobe FW, Kerlan RK. Shoulder impingement syndrome in athletes treated by an anterior acromioplasty.

13 Jobe CM. Evidence for superior glenoid impingement upon the rotator cuff. F Shoulder Elbow Surg 1993;2:51-4.

14 Burkehead WZ. The biceps tendon. In: Rockwood CA, Matsen FA, eds. The shoulder. Philadelphia: WB Saunders, 1990:791

15 Blazina ME, Satzman JS. Recurrent anterior subluxation of the shoulder in athletes-a distinct entity. In: Proceedings of the American Academy of Orthopaedic Surgeons. $\mathcal{F}$ Bone foint Surg Am 1969;51A:1037-8.

6 Litchfield R, Hawkins R, Dillman CJ, Atkins J, Hagerman GB. Rehabilitation for the overhead athlete. $\mathcal{F}$ Orthop Sports Phys Ther 1993;18:433-41. 
17 Wilk KE Arrig C J Current concepts in the rehabilitation of the athletic shoulder. F Orthop Sports Phys Ther 1993;18:365-78.

18 Dines DM, Levinson M. The conservative management of the unstable shoulder including rehabilitation. Clin Sports Med 1995;14:797-816.

19 Bigliani LU, Kurzweil PR, Schwartzbach CC, Wolfe IN, Flatow EL. Inferior capsular shift procedure for anteriorinferior shoulder instability in athletes. $A m \mathcal{F}$ Sports $M e d$ 1994;22:578-84.

20 Altchek DE, Warren RF, Wickiewicz TL, Ortiz G. Arthroscopic labral debridement: a three year follow up study. Am ₹ Sports Med 1992;20:702-6.

21 Terry G, Friedman SJ, Uhl TL. Arthroscopically treated tears of the glenoid labrum: factors affecting outcome. $A m$ tears of the glenoid labrum: facto

22 Martin DR, Garth WP. Results of arthroscopic debridement of glenoid labral tears. Am $\mathcal{f}$ Sports Med 1995;23:447-51.

23 Rowe CR, Patel D, Southmayd WW. The Bankar procedure: a long term end result study. $\mathcal{f}$ Bone foint Surg

24 Lombardo SJ, Kerlan RK, Jobe FW. The modified Bristow procedure for recurrent dislocation of the shoulder. $\mathcal{F}$ Bone foint Surg Am 1976;58A:256-61.

25 Altchek DW, Warren RF, Skyhar MJ. T-plasty modification of the Bankart procedure for multidirectional instability of the anterior and inferior types. F Bone foint Surg Am 1991; 73A:105-12.

26 Jobe FW, Giangarra CE, Kvitne RS, Glousman RE. Anterior capsulolabral reconstruction of the shoulder in athletes in overhand sport. Am $¥$ Sports Med 1991;19:428-34.

27 Altchek DW, Warren RF, Skyhar MJ. T-plasty modification of the Bankart procedure for multidirectional instability of of the Bankart procedure for multidirectional instability of
the anterior and inferior types. $\mathcal{F}$ Bone foint Surg Am 1991; the anterio

28 Copeland $S$. Throwing injuries of the shoulder. Br $\mathcal{F}$ Sports Med 1993;27:221.

\section{Society of Apothecaries of London Diploma in Sports Medicine}

Examinations in 1997: Written papers June 4 Clinical/Oral June 5

Closing date for applications 6 weeks before the examinations

\section{Diploma in Musculoskeletal Medicine}

Examinations in 1997: Written papers March 3

Clinical and Oral March 12

Closing date for applications January 20

Further details, syllabus etc from:

Society of Apothecaries of London

Black Friars Lane

London EC4V 6EJ 